\title{
Quantum signatures of competing electron trajectories in antidot superlattices
}

\author{
J. Schluck, ${ }^{1, *}$ J. Feilhauer, ${ }^{2,3}$ K. Pierz, ${ }^{3}$ H. W. Schumacher, ${ }^{3}$ D. Kazazis, ${ }^{4,5}$ U. Gennser, ${ }^{5}$ and T. Heinzel ${ }^{1, \dagger}$ \\ ${ }^{1}$ Condensed Matter Physics Laboratory, Heinrich-Heine-Universität, D-40204 Düsseldorf, Germany \\ ${ }^{2}$ Institute of Electrical Engineering, Slovak Academy of Sciences, 84104 Bratislava, Slovakia \\ ${ }^{3}$ Physikalisch-Technische Bundesanstalt, Bundesallee 100, D-38116 Braunschweig, Germany \\ ${ }^{4}$ Paul Scherrer Institut, Villigen-PSI, 5232, Switzerland \\ ${ }^{5}$ Centre de Nanosciences et de Nanotechnologies (C2N), CNRS, Univ. Paris Sud-Université Paris-Saclay, 91120 Palaiseau, France
}

(Received 18 September 2017; revised manuscript received 30 August 2018; published 9 October 2018)

\begin{abstract}
Magnetotransport measurements on antidot lattices with square and hexagonal geometries are reported. The resistivity shows a superposition of the classical commensurability resonances and Shubnikov-de Haas type oscillations. In this range of magnetic fields, quantum Hall plateaus are already developed, but shifted to lower fields. The transitions between these plateaus are found to be in-phase with an additional small-amplitude oscillation in the resistivity. This is interpreted with the help of numerical simulations as a quantum signature of the competition between skipping and hopping transport in antidot superlattices.
\end{abstract}

DOI: 10.1103/PhysRevB.98.165415

\section{INTRODUCTION}

Antidot superlattices in two-dimensional electron gases (2DEGs) have been an active field of research since their first implementations. Originally studied mostly in $\mathrm{Ga}[\mathrm{Al}] \mathrm{As}$ heterostructures [1-3], more recent experiments focused on novel materials such as graphene [4,5], topological insulators [6,7], and black phosphorus [8]. In the semiclassical regime, which can be characterized by a lattice constant $a$ significantly larger than the electron Fermi wavelength $\lambda_{F}$ but small compared to the mean free path $\ell$ of the electrons in the absence of the superlattice, the longitudinal magnetoresistivity $\rho_{x x}(B)$ shows prominent commensurability resonances, which demonstrates the inadequacy of the Boltzmann model that predicts $\rho_{x x}$ to be independent of the applied perpendicular magnetic field $B$. Rather, the dominant scattering takes place at the superlattice potential, and neither the location nor the shape and strength of the scatterers is random. This correlated scattering leads to a mixed phase space for the conduction electrons, where regular and chaotic regions coexist, the relevance of which depends on the experimental parameters $[9,10]$.

As an extension to these classical effects, superimposed quantum oscillations have been observed in several experiments [11-15]. They can be modeled semiclassically using the periodic orbit theory [16,17], but also fully quantummechanical calculations have been reported [18-21]. In case they are $B$-periodic, they indicate electronic interferences along trajectories that are commensurate with the superlattice potential but independent of $B$ within a certain magnetic field interval [11-13,16,17]. $1 / B$-periodic quantum oscillations, on the other hand, are attributed to Landau quantization where the electron trajectories are cyclotron orbits that remain essentially undisturbed by the superlattice potential [15]. The type of periodicity may furthermore depend on the magnetic

\footnotetext{
*jakob.schluck@hhu.de

†thomas.heinzel@hhu.de
}

field. For example, Weiss et al. have reported $1 / B$-periodicity for magnetic fields where the cyclotron orbit fits in the area in between the antidots, and $B$-periodicity for smaller magnetic fields [11]. In this way, antidot lattices have also been test systems for the relation between classical trajectories and the quantum properties of the system. The contributions to the conductivity are often grouped as being related to either scattering or band transport. In a classical picture, the former describes a hopping between localized states and is therefore known as a hopping contribution, while the latter is associated with extended states in the antidot lattice that skip along lines of antidots, sometimes also referred to as runaway trajectory [22]. The relevance of each contribution depends on the sample parameters and the magnetic-field range. An instructive demonstration was reported for rectangular lattices [23-25], where electrons were found to skip along the short lattice direction and hop along the long one.

In the present work, we demonstrate experimentally how hopping and skipping transport contributes to the conductivity in square and hexagonal antidot lattices. Our samples are in a regime where quantum signatures of both transport mechanisms coexist in the magnetoresistance over a wide magnetic-field range. Their influence can also be seen in the Hall resistance where quantized plateaus are shifted to lower magnetic fields as compared to the classical extrapolation. This can be attributed to the selective backscattering of single edge states via skipping orbits. In Sec. II, we present our experimental setup and the details on the sample preparation. Our experimental findings are discussed in Sec. III and interpreted with the help of numerical simulations in Sec. IV. The paper ends with a conclusion in Sec. V.

\section{SAMPLE PREPARATION AND EXPERIMENTAL SETUP}

A $\mathrm{Ga}_{0.7} \mathrm{Al}_{0.3} \mathrm{As}$ heterostructure with a $2 \mathrm{DEG}$ formed $90 \mathrm{~nm}$ below the surface is used. After infrared illumination, the pristine 2DEG has a density of $n=4.0 \times 10^{15} \mathrm{~m}^{-2}$ and 

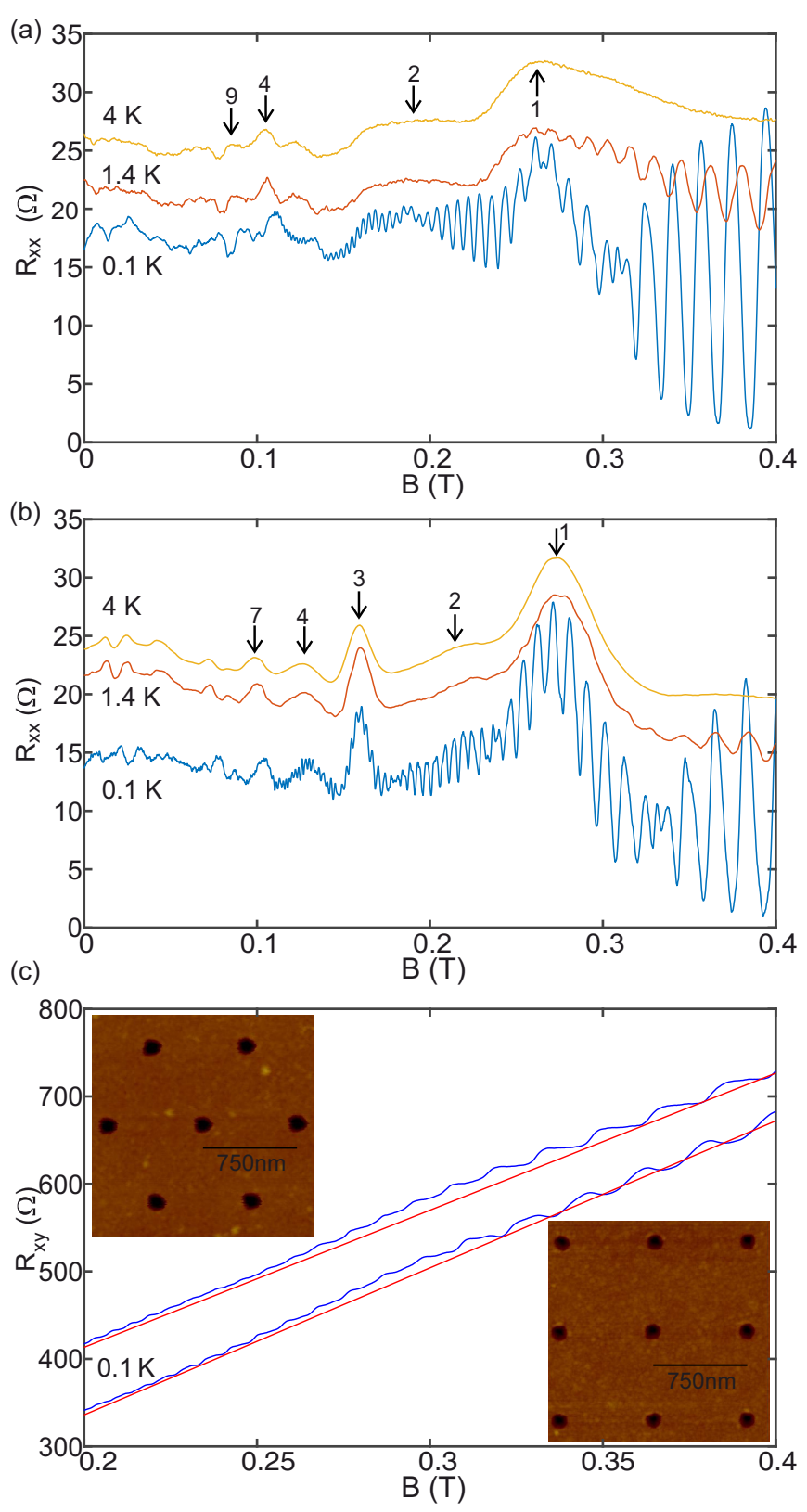

FIG. 1. (a) $R_{x x}(B)$ of the sample with square symmetry at several temperatures. (b) $R_{x x}(B)$ of the sample with hexagonal symmetry at several temperatures. Numbers label the resistance maxima by the number of antidots encircled at the given magnetic field. The traces at elevated temperatures are vertically offset for clarity. (c) Insets: AFM pictures of the two samples. The lattice constant $a$ is $750 \mathrm{~nm}$ in both cases. Main figure: Hall resistance for both sample types at base temperature. The hexagonal trace is vertically offset by $100 \Omega$ for clarity. The classical extrapolation is shown in red.

a mobility of $890 \mathrm{~m}^{2} / \mathrm{V}$ s at liquid helium temperature, corresponding to a mean free path of $92 \mu \mathrm{m}$. Antidot lattices with square and hexagonal symmetries are prepared by electron beam lithography and subsequent reactive ion etching. A lattice constant of $a=750 \mathrm{~nm}$ and a lithographic antidot diameter of $d_{\text {lith }}=150 \mathrm{~nm}$ are used for both geometries; cf. Fig. 1(c) for atomic force microscope (AFM) images. The etching profile is rather shallow, such that a depletion of the
2DEG is only given in the central region of each individual antidot [26]. A reasonable approximation of the resulting electronic antidot diameter $d$ is obtained by comparison to numerical simulations and is given below. The resulting aspect ratio $d / a$ thus lies in the range $d / a<0.2$. The lattices extend over $250 \mu \mathrm{m}$ in length and $100 \mu \mathrm{m}$ in width, which is also the width of the Hall bar. The spacing between the voltage probes is $150 \mu \mathrm{m}$. The samples are transferred to a dilution refrigerator with a base temperature of $25 \mathrm{mK}$ and an electron temperature estimated to be $0.1 \mathrm{~K}$. Measurements at elevated temperatures are performed in a helium gas flow cryostat with a variable temperature insert. The diagonal and Hall resistance are determined by applying a low-frequency ac current and measuring the voltage drop across the Hall bar with a lock-in amplifier.

\section{EXPERIMENTAL RESULTS}

In Fig. 1, we show the results of the magnetoresistance measurements. For both the square [Fig. 1(a)] and the hexagonal [Fig. 1(b)] lattice, the overall resistance is of the order of $20 \Omega$, which corresponds to a mean free path that is much larger than the antidot lattice constant. Similar behavior was observed previously in systems consisting of antidot lattices with a high aspect ratio in high-mobility heterostructures [27]. A series of pronounced commensurability oscillations (COs) is observed, with the principal peak at a magnetic field around $270 \mathrm{mT}$. The numbers labeling the resistance maxima in the figure indicate the number of antidots that are encircled at the given magnetic field. Superimposed to this, a short-period oscillation emerges at about $100 \mathrm{mT}$ at $0.1 \mathrm{~K}$. It gets rapidly suppressed as one increases the temperature and has a $1 / B$ periodicity. It is straightforward to interpret it as a type of Shubnikov-de Haas ( $\mathrm{SdH}$ ) oscillation. Spin-splitting becomes visible for magnetic fields $B \geqslant 700 \mathrm{mT}$ and will therefore be neglected in the following. For some magnetic fields, deviations from the periodicity can be observed. For example, in the hexagonal lattice at $330 \mathrm{mT}$ and at $300 \mathrm{mT}$ in the square lattice, there appears an additional maximum in the resistance accompanied by a suppression of the amplitude of the neighboring peaks. At lower magnetic fields, additional distortions can be seen in both amplitude and periodicity. In Fig. 1(c), the Hall effect for both samples is shown. From a comparison of the classical extrapolation to the data, one can see that for low magnetic fields $(B \leqslant 250 \mathrm{mT})$ the modification induced by the antidots is negligible. For magnetic fields beyond the principal $\mathrm{CO}$, quantum Hall signatures are found and the average slope of the data coincides with the classical extrapolation. In the magnetic-field range of the principal $\mathrm{CO}$, however, the Hall resistance is increased with respect to the classical extrapolation, although plateaus are already formed.

We proceed by studying the interplay of diagonal and Hall resistance. For pristine 2DEGs, the maxima in the SdH oscillations coincide with the transitions between quantum Hall plateaus. This can be understood in terms of the formation of extended states [28]. To check this connection in our system, we numerically take the derivative of the Hall resistance with respect to the magnetic field $d R_{x y} / d B$ and compare it to the diagonal resistance. The result is shown in Figs. 2(a) and 2(b) for the square and hexagonal lattice, respectively. Here we 

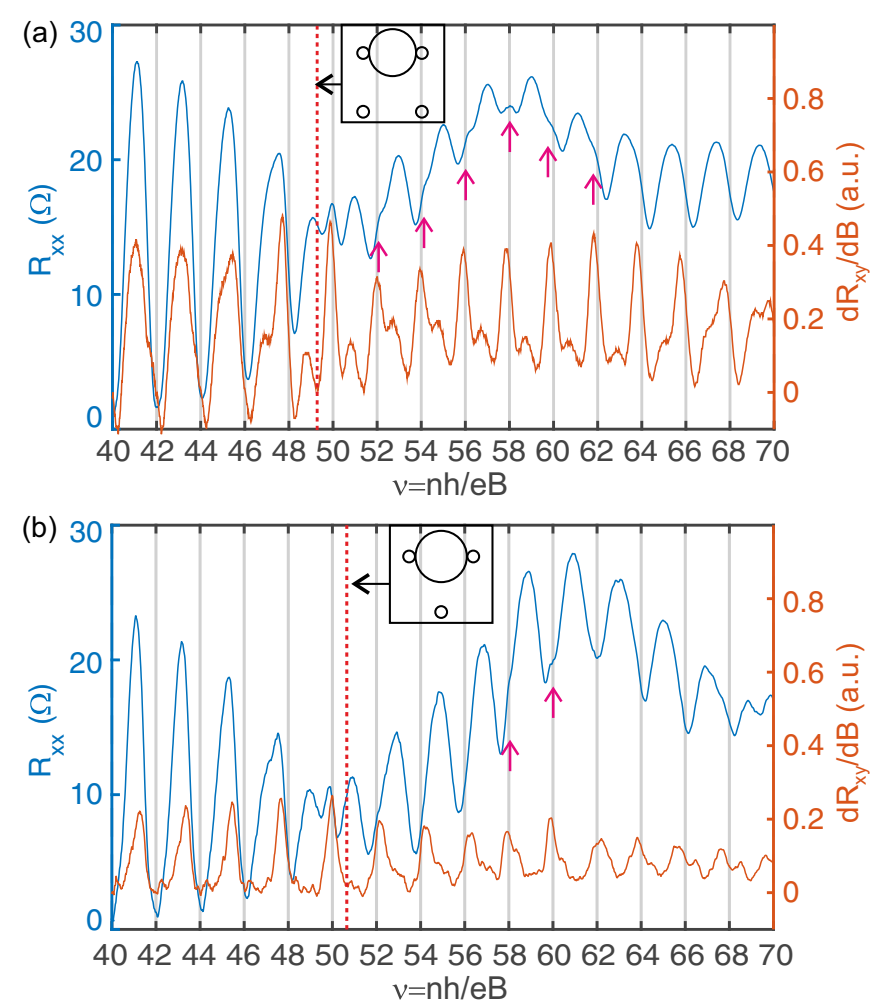

FIG. 2. Comparison of the diagonal resistance $R_{x x}$ and the derivative of the Hall resistance $d R_{x y} / d B$ for the square (a) and hexagonal (b) lattice as a function of the filling factor $v=h n / e B$. The pink arrows indicate the magnetic fields where significant deviations of the regular $\mathrm{SdH}$ oscillations are visible. The insets show the filling factor where skipping orbits become possible in a classical picture.

have plotted the data against the spin-resolved Landau-level filling factor $v=h n / e B$. In this representation it becomes visible that in fact the superimposed quantum oscillation has a more complex structure. Besides a dominant large-amplitude oscillation, there is also a small-amplitude oscillation visible (marked by the pink arrows), which is out of phase with the former one. It is more prominent in the case of the square lattice, but also visible in the hexagonal lattice. For a pristine $2 \mathrm{DEG}$ one can expect the maxima in $R_{x x}$ and $d R_{x y} / d B$ to occur at odd integers, corresponding to half-filled spin-degenerate Landau levels. For magnetic fields beyond the principal CO, i.e., $v \leqslant 48$, this is also what we observe in our samples. Interestingly, the situation changes for lower magnetic fields. For filling factors $v \geqslant 50$, the resistance maxima in $R_{x x}$ still coincide with odd filling factors, but the maxima in $d R_{x y} / d B$ can be found at even integers. This oscillation is now in-phase with the small-amplitude oscillation observed in $R_{x x}$.

\section{INTERPRETATION AND NUMERICAL SIMULATIONS}

Previously, quantum effects in antidot superlattices were often interpreted semiclassically $[16,17]$. The framework of periodic orbit theory allows a calculation of the contributions to the density of states (DOS) stemming from various periodic orbits. From this, the emergence of quantum oscillations and their periods was predicted.

In our case, such a treatment is not justified. Our sample design leads to a situation in which the potential outside of the antidots is almost constant and can therefore not stabilize periodic orbits. Therefore, the previously reported oscillations caused by electron cyclotron motion being strongly modified $[11,13]$, observed for antidot lattices with a large $d / a$ ratio, are expected to play only a minor role in our case. The only significant periodic orbits remaining are the ones where the electron undergoes cyclotron motion undisturbed by the superlattice. The effects observed by us are also unlikely to originate from the Hofstadter butterfly [29-32]. Such effects are periodic in $\phi / \phi_{0}=B A e / h$, where $A$ is the size of the unit cell of the superlattice, in contrast to the $1 / B$-periodicity seen here.

Instead we propose an explanation in terms of the relation between the DOS and $\rho_{x x}$, which can be connected to skipping and hopping transport as used for a description within a classical picture. We first take a look at the elements of the magnetoconductivity tensor. Assuming isotropic transport, we have $\sigma_{x x}=\frac{\rho_{x x}}{\rho_{x x}^{2}+\rho_{x y}^{2}}$ and $\sigma_{x y}=\frac{-\rho_{x y}}{\rho_{x x}^{2}+\rho_{x y}^{2}}$. For the whole range of interest here $(0.1<B<0.5 \mathrm{~T})$, the Hall conductivity is at least one order of magnitude larger than the diagonal one. We can conclude $\sigma_{x x} \approx \rho_{x x} / \rho_{x y}^{2}$, which implies maxima in resistivity to coincide with maxima in conductivity. This is in contrast to earlier works by Ishizaka et al. [21,33], who reported changes in the interplay of resistivity and DOS over the course of the COs, but in their system $\sigma_{x x} \approx \sigma_{x y}$ and thus the discussed structure was caused by the Hall conductivity.

More relevant to our situation is the work by Schuster et al., who studied rectangular antidot lattices [23] and found a magnetic-field range where quantum oscillations were present in $\rho_{x x}$ that were out of phase with those in $\rho_{y y}$. Neudert et al. explained this in terms of transport being dominated by skipping along the short period of the lattice and by hopping between localized states along the long period [24]. In this picture, the conductance by hopping is enlarged at the maxima in the density of states, where many localized states exist. The electrons then move inside the lattice via a so-called hopping conductivity, i.e., by being scattered from one localized state to another. In contrast to this, the skipping along a line of antidots is identified as a band transport mechanism. The associated group velocity and thus also the contribution to the conductivity was found to be enhanced for situations in which there are few localized states. Intuitively one can consider the skipping orbits to carry current most efficiently when there are no localized states for them to scatter into; therefore, their contribution to the conductivity is strong for a low density of states.

To interpret our data, we employ numerical simulations based on the KWANT transport package [34]. KWANT allows for the implementation of finite tight-binding scattering regions connected to semi-infinite leads, which models our Hall bar structure connected to voltage probes. It gives access to various transport properties, like the transmission between the contacts, which can be converted to a resistance via the Landauer-Büttiker formalism [35]. We calculate the fourterminal resistance [36] as well as the DOS of a $10 \times 8 \mu \mathrm{m}^{2}$ 

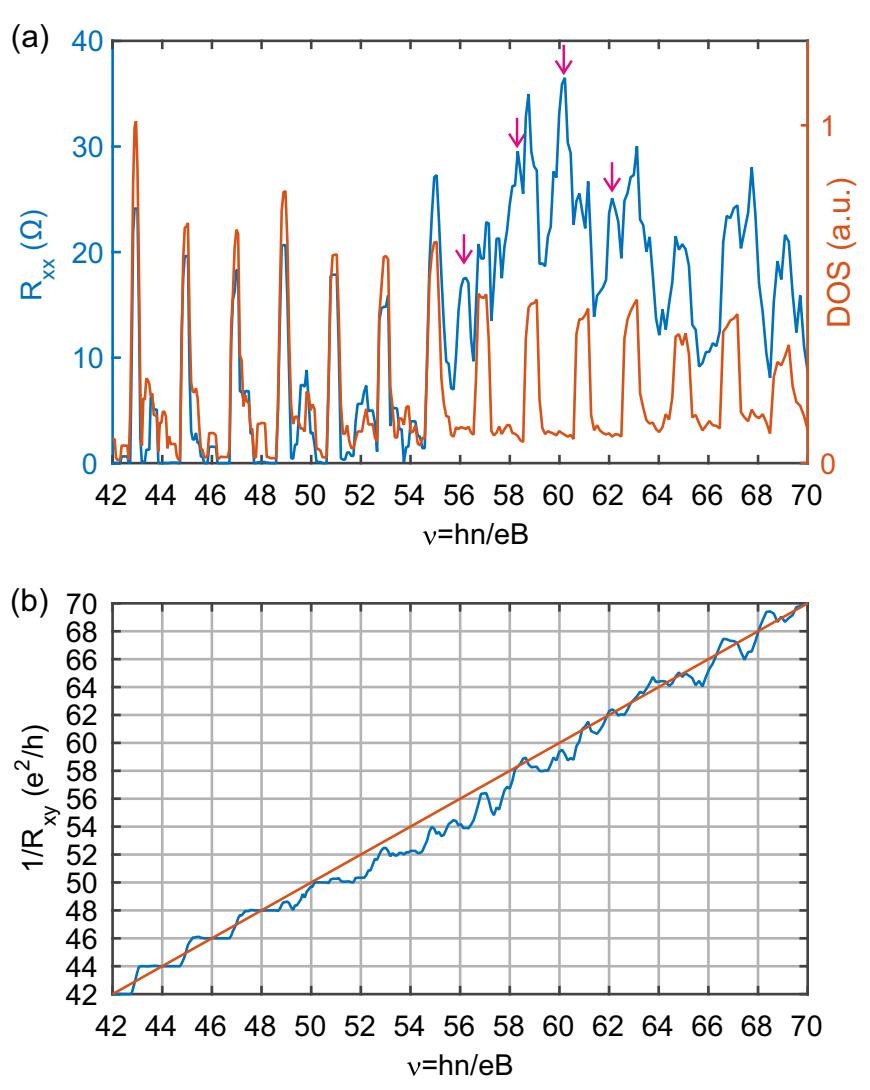

FIG. 3. (a) Fully quantum-mechanical calculation of the magnetoresistance (blue) and the density of states (red) of a square antidot lattice as a function of the filling factor at $T=0$ based on the KWANT package. Pink arrows exemplify resistance maxima that are out of phase with the DOS. (b) Calculated inverse Hall resistance (blue) and the classical extrapolation (red) as a function of the filling factor. For filling factors $48 \leqslant v \leqslant 62$ the quantized plateaus are shifted to lower magnetic fields, whereas they recover their conventional behavior for stronger fields.

scattering region. Larger arrays are beyond the scope of the present work due to technical limitations. A tight-binding lattice constant of $4 \mathrm{~nm}$ and Fermi energy corresponding to the density of our 2DEG were used. A square-lattice antidot potential is implemented via the on-site potential

$$
V(x, y)=\frac{3}{2} E_{F}\left(\sin \frac{\pi x}{a} \sin \frac{\pi y}{a}\right)^{\beta},
$$

where $E_{F}$ is the Fermi energy. The steepness parameter is set to $\beta=64$ reflecting the low value of $d / a$ in our samples. The parameters were chosen to roughly reproduce the measured resistance, but the qualitative behavior is robust to the exact values. They correspond to an electronic diameter of $d \approx$ $50 \mathrm{~nm}$ at the Fermi energy. This reflects the shallow etching in the sample, which leads to a depletion of the 2DEG in an area smaller than the lithographic one. The magnetic field was sampled with a resolution of $0.25 \mathrm{mT}$. The simulation does not take thermal smearing into account, but its effect is emulated by numerical smoothing of the results. Scattering at residual impurities is included via random on-site disorder.

In Fig. 3(a) the calculated resistance and DOS are shown as a function of the filling factor in the range of the principal
CO. The position of the classical resistance peak around filling factor 60 is well reproduced. Additional oscillations with varying amplitudes are superimposed. In the DOS a series of well-developed peaks, which are periodic in $1 / B$, can be observed. They appear at odd-integer filling factors, so the positions of the Landau levels are not shifted by the superlattice potential, which is in contrast to earlier findings concerning electric [37,38] and magnetic [39,40] unidirectional superlattices. For each peak in the DOS, a corresponding peak in the resistance can be seen, indicating an origin from hopping transport. These peaks are thus equivalent to the conventional SdH oscillations. Additional resistance maxima with comparable amplitude (see pink arrows in the figure) appear for low DOS values. We interpret them as being caused by band transport.

The calculated inverse Hall resistance is shown in Fig. 3(b). Here we also find semiquantitative agreement with the experimental data. For filling factors $48 \leqslant v \leqslant 62$, a clear shift of the Hall plateaus away from the classical extrapolation is observed. For lower as well as higher magnetic fields, the conventional behavior of a pristine $2 \mathrm{DEG}$ is recovered.

Transferring these numerical results to our experiment, we thus identify the large-amplitude quantum oscillation as conventional $\mathrm{SdH}$ oscillations, since they appear at oddinteger filling factors and there is no indication of a shift of the DOS. We conclude that the dominant contribution to the conductivity, and thus also the resistivity within the principal $\mathrm{CO}$, is caused by hopping transport. Band transport is only responsible for the smaller corrections of the diagonal conductivity, manifested in the formation of additional resistivity peaks with reduced amplitude. In contrast, the Hall resistance is profoundly affected by band transport. The decoupling of the transitions between the plateaus from the peaks in the DOS indicates that hopping transport cannot be responsible for the observed increase in Hall resistance. This raises the question of how quantization can be maintained, since clearly the number of edge states entering the array from the leads must be the same as in the pristine 2DEG. To clarify this issue, we have employed KWANT to calculate the propagation of waves emitted from one contact inside the sample. For this,

$$
\left|\Psi_{j}\right|^{2}=\sum_{i}\left|\psi_{i j}\right|^{2}
$$

is calculated, where $\psi_{i}$ is the wave function in the sample area emerging from the $i$ th mode in lead $j$. The result is shown in Fig. 4 for a magnetic field of $B=289 \mathrm{mT}$, where the longitudinal resistance is at a minimum and the Hall resistance is one plateau above the one expected for this filling factor. One clearly sees how the majority of the modes is directly absorbed by the next voltage probe starting at $x=-4 \mu \mathrm{m}$. The outermost edge state, however, is crossing the Hall bar via a skipping motion along a line of antidots and is reflected back into the emitting contact. In this way, the extended state formed in the center of a Landau level is not needed to equilibrate the Hall voltage, and we can explain the shift of the Hall plateaus away from the classical extrapolation as caused by a band transport effect. For stronger magnetic fields $(B \geqslant 0.35 \mathrm{~T})$, the transitions in the Hall effect coincide with DOS maxima and odd filling factors again. In a classical 


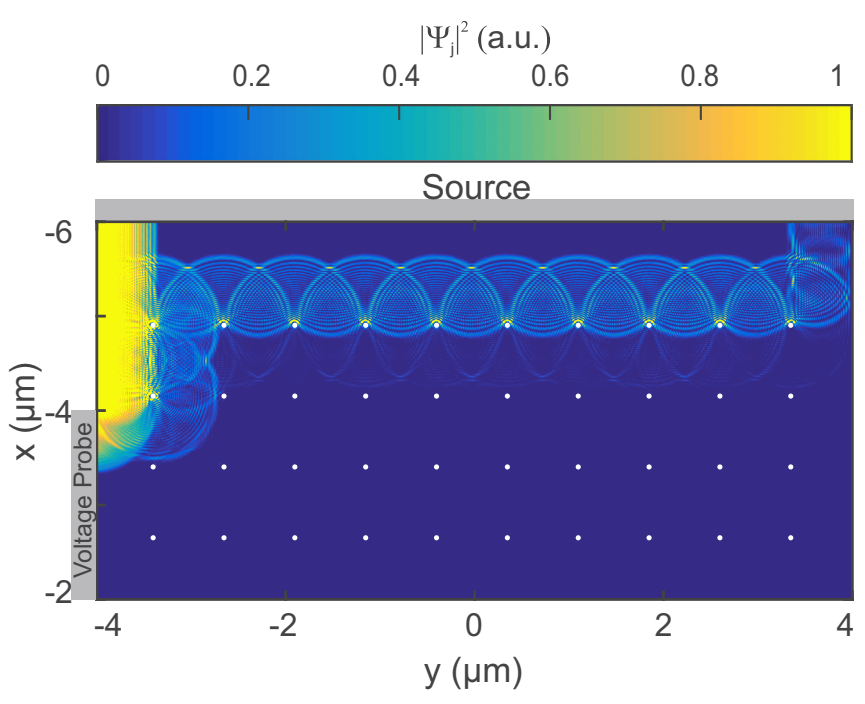

FIG. 4. The sum over the square of the absolute values of the wave functions of all modes entering the Hall bar from the source contact. A magnetic field of $B=289 \mathrm{mT}$ pointing out of the direction of the plane is applied. The white circles correspond to the diameter of the antidots at the Fermi energy. The majority of the modes is drained by the first voltage probe, starting at $-4 \mu \mathrm{m}$, but the outermost edge state gets directly backscattered via a skipping orbit.

picture, this corresponds to the magnetic field range where the condition $2 R_{c}<a-d$ is fulfilled, and it is readily interpreted as the vanishing of skipping orbits. Electrons can no longer travel along a line of antidots, but are rather localized to closed orbits either around antidots or in the void space between them. Interestingly, the relative increase of the Hall resistance observed here is in contrast to earlier findings by Haug et al. [41], where randomly positioned repulsive scatterers lead to a relative decrease in the Hall resistance. This underlines the importance of the regular arrangement of the antidots. An analysis in terms of electron trajectories, similar to the one presented here, was done by Schuster et al. [42] and Tsukagoshi et al. [43]. In both studies, the authors varied the diameter of the antidots experimentally and could thereby tune the relative contributions of hopping and skipping transport to the classical COs. Lüthi et al. [25] described the influence of both transport mechanisms in terms of trajectories in rectangular lattices. They clearly state the coexistence of pinned and skipping orbits in their lattice, but, in contrast to our findings, the quantum contributions of each type of orbit affect either only $\sigma_{x x}$ or only $\sigma_{y y}$.

\section{SUMMARY AND CONCLUSIONS}

We have demonstrated experimentally the coexistence of quantum signatures of hopping and skipping transport in antidot superlattices with low aspect ratio defined in 2DEGs within both the longitudinal and the Hall resistivity. Over the course of the main commensurability oscillation, we observe a decoupling of the transitions between quantum Hall plateaus from the longitudinal conductivity maxima. We propose an explanation in terms of extended skipping orbits, which are related to band transport. They are accompanied by additional minor longitudinal conductivity maxima, whereas the major ones are related to hopping transport. We substantiate our interpretation by a comparison to a fully quantum-mechanical numerical simulation based on the KWANT package, where we find semiquantitative agreement with the experiment. For increased magnetic fields, where the cyclotron radius is smaller than the antidot lattice constant, the behavior of a pristine 2DEG is recovered, which is in agreement with simple semiclassical considerations.

\section{ACKNOWLEDGMENTS}

The authors would like to thank K. Richter and A. Endo for valuable discussions. J.S. and T.H. gratefully acknowledge financial support from Heinrich Heine University Düsseldorf. J.F. is thankful for the grant VEGA 2/0162/18.
[1] D. Weiss, M. L. Roukes, A. Menschig, P. Grambow, K. von Klitzing, and G. Weimann, Phys. Rev. Lett. 66, 2790 (1991).

[2] K. Ensslin and P. M. Petroff, Phys. Rev. B 41, 12307 (1990).

[3] A. Lorke, J. P. Kotthaus, and K. Ploog, Phys. Rev. B 44, 3447 (1991).

[4] R. Yagi, R. Sakakibara, R. Ebisuoka, J. Onishi, K. Watanabe, T. Taniguchi, and Y. Iye, Phys. Rev. B 92, 195406 (2015).

[5] A. Sandner, T. Preis, C. Schell, P. Giudici, K. Watanabe, T. Taniguchi, D. Weiss, and J. Eroms, Nano Lett. 15, 8402 (2015).

[6] M. Song, J.-H. Chu, J. Zhou, S. Tongay, K. Liu, J. Suh, H. Chen, J. S. Kang, X. Zou, and L. You, Nanotechnology 26, 265301 (2015).

[7] H. Maier, J. Ziegler, R. Fischer, D. Kozlov, Z. D. Kvon, N. Mikhailov, S. A. Dvoretsky, and D. Weiss, Nat. Commun. 8, 2023 (2017).
[8] A. Cupo, P. M. Das, C.-C. Chien, G. Danda, N. Kharche, D. Tristant, M. Drndić, and V. Meunier, ACS Nano 11, 7494 (2017).

[9] R. Fleischmann, T. Geisel, and R. Ketzmerick, Phys. Rev. Lett. 68, 1367 (1992).

[10] R. Fleischmann, T. Geisel, and R. Ketzmerick, Europhys. Lett. 25, 219 (1994).

[11] D. Weiss, K. Richter, A. Menschig, R. Bergmann, H. Schweizer, K. von Klitzing, and G. Weimann, Phys. Rev. Lett. 70, 4118 (1993).

[12] R. Schuster, K. Ensslin, D. Wharam, S. Kühn, J. P. Kotthaus, G. Böhm, W. Klein, G. Tränkle, and G. Weimann, Phys. Rev. B 49, 8510 (R) (1994).

[13] F. Nihey, S. W. Hwang, and K. Nakamura, Phys. Rev. B 51, 4649 (1995).

[14] Y. Iye, M. Ueki, A. Endo, and S. Katsumoto, J. Phys. Soc. Jpn. 73, 3370 (2004). 
[15] Y. Kato, A. Endo, S. Katsumoto, and Y. Iye, Phys. Rev. B 86, 235315 (2012).

[16] K. Richter, Europhys. Lett. 29, 7 (1995).

[17] G. Hackenbroich and F. van Oppen, Europhys. Lett. 29, 151 (1995).

[18] D. Pfannkuche and R. R. Gerhardts, Phys. Rev. B 46, 12606 (1992).

[19] R. B. S. Oakeshott and A. MacKinnon, J. Phys.: Condens. Matter 6, 1519 (1994).

[20] H. Silberbauer and U. Rössler, Phys. Rev. B 50, 11911 (1994).

[21] S. Ishizaka, F. Nihey, K. Nakamura, J. Sone, and T. Ando, Phys. Rev. B 51, 9881 (1995).

[22] E. Baskin, G. Gusev, Z. Kvon, A. Pogosov, and M. Entin, JETP Lett. 55, 678 (1992).

[23] R. Schuster, K. Ensslin, J. P. Kotthaus, G. Böhm, and W. Klein, Phys. Rev. B 55, 2237 (1997).

[24] R. Neudert, P. Rotter, U. Rössler, and M. Suhrke, Phys. Rev. B 55, 2242 (1997).

[25] S. Lüthi, T. Vancura, K. Ensslin, R. Schuster, G. Böhm, and W. Klein, Phys. Rev. B 55, 13088 (1997).

[26] See supplemental material at http://link.aps.org/supplemental/ 10.1103/PhysRevB.98.165415 for a SEM picture of a single antidot along a cleaved edge.

[27] Z. Q. Yuan, C. L. Yang, R. R. Du, L. N. Pfeiffer, and K. W. West, Phys. Rev. B 74, 075313 (2006).

[28] Introduction to the Theory of the Integer Quantum Hall Effect, edited by J. Hajdu (Wiley-VCH, Weinheim, 1994).
[29] D. Hofstadter, Phys. Rev. B 14, 2239 (1976).

[30] F. H. Claro and G. H. Wannier, Phys. Rev. B 19, 6068 (1979).

[31] T. Schlösser, K. Ensslin, J. P. Kotthaus, and M. Holland, Europhys. Lett. 33, 683 (1996).

[32] M. C. Geisler, J. H. Smet, V. Umansky, K. von Klitzing, B. Naundorf, R. Ketzmerick, and H. Schweizer, Phys. Rev. Lett. 92, 256801 (2004).

[33] S. Ishizaka and T. Ando, Phys. Rev. B 56, 15195 (1997).

[34] C. W. Groth, M. Wimmer, A. R. Akhmerov, and X. Waintal, New J. Phys. 16, 063065 (2014).

[35] M. Büttiker, Phys. Rev. Lett. 57, 1761 (1986).

[36] H. U. Baranger and A. D. Stone, Phys. Rev. B 40, 8169 (1989).

[37] M. Tornow, D. Weiss, A. Manolescu, R. Menne, K. v. Klitzing, and G. Weimann, Phys. Rev. B 54, 16397 (1996).

[38] A. Endo and Y. Iye, J. Phys. Soc. Jpn. 77, 054709 (2008).

[39] K. W. Edmonds, B. L. Gallagher, P. C. Main, N. Overend, R. Wirtz, A. Nogaret, M. Henini, C. H. Marrows, B. J. Hickey, and S. Thoms, Phys. Rev. B 64, 041303 (2001).

[40] J. Shi, F. M. Peeters, K. W. Edmonds, and B. L. Gallagher, Phys. Rev. B 66, 035328 (2002).

[41] R. J. Haug, R. R. Gerhardts, K. von Klitzing, and K. Ploog, Phys. Rev. Lett. 59, 1349 (1987).

[42] R. Schuster, G. Ernst, K. Ensslin, M. Entin, M. Holland, G. Böhm, and W. Klein, Phys. Rev. B 50, 8090 (1994).

[43] K. Tsukagoshi, T. Nagao, M. Haraguchi, S. Takaoka, K. Murase, and K. Gamo, Superlattices Microstruct. 23, 493 (1998). 